\title{
El efecto de las patentes en la innovación ambiental: Balances y retos
}

\section{The effect of patents on environmental innovation: perspectives and challenges}

\author{
DOI: https://doi.org/10.17981/juridcuc.16.1.2020.07
}

Fecha de Recepción: 13/09/2019. Fecha de Aceptación: 05/12/2019

Iván Vargas-Chaves

Universidad Militar Nueva Granada, Bogotá (Colombia)

ivan.vargas@unimilitar.edu.co

Omar Clavijo-Bernal

Universidad Nacional de Colombia, Bogotá (Colombia)

ofclavijob@unal.edu.co

\author{
Jonathan Sánchez-Rippe (i) \\ Universidad del Rosario, Bogotá (Colombia) \\ jonathand.sanchez@urosario.edu.co
}

Para citar este artículo:

Vargas-Chaves, I.,Clavijo-Bernal, O. y Sánchez-Rippe, J. (2020). El efecto de las patentes en la innovación ambiental: Balances y retos. JURÍDICAS CUC, 16(1). 177-196. DOI: http://dx.doi. org/10.17981/juridcuc.16.1.2020.07

\section{Resumen}

El artículo busca resaltar el papel adquirido por las patentes en el ámbito de las invenciones y modelos de utilidad que pueden ser considerados innovaciones ambientales. Su objetivo es caracterizar el rol clave que han tenido a través de los nuevos productos, procesos y servicios que aportan soluciones de sostenibilidad, generan impactos positivos al ambiente o mitigan efectos negativos derivados de aquellas actividades que contribuyen a las actuales condiciones de deterioro ambiental del planeta. A través de un enfoque metodológico deductivo, los autores parten de nociones generales sobre propiedad intelectual, ambiente y desarrollo, para así llegar a reflexiones propias a manera de hallazgos, y situar a las patentes sobre innovaciones ambientales como un eslabón clave en pro del ambiente, evidenciando así, como en efecto, las patentes han logrado aportar al mejoramiento de las condiciones ambientales, ayudando al Estado y a las empresas al cumplimiento de sus compromisos en materia ambiental con la sociedad.

Palabras clave: Ecoinnovación; innovación ambiental; patentes; políticas ambientales; propiedad intelectual; Sostenibilidad

\begin{abstract}
The article seeks to highlight the role acquired by patents in the field of inventions and utility models that can be considered environmental innovations. Its objective is to characterize the key role that they have had through new products, processes and services that provide solutions of sustainability, generate positive impacts to the environment or mitigate negative effects derived from those activities that contribute to the current conditions of environmental deterioration of the planet. Through a deductive methodological approach, the authors start from general notions about intellectual property, environment and development, in order to arrive at their own reflections as findings, and to place patents on environmental innovations as a key link in favor of the environment, thus showing how, in effect, patents have managed to contribute to the improvement of environmental conditions, helping the State and companies to comply with their environmental commitments to society.
\end{abstract}

Keywords: Ecoinnovation; environmental innovation; environmental policy; entellectual property; patents; sustainability 


\section{INTRODUCCIÓN}

La innovación en materia ambiental ha adquirido un papel protagónico en nuestra sociedad como solución a las condiciones de deterioro ambiental generadas por el hombre desde la revolución industrial. Tras comprender los daños irreversibles ocasionados por éste, se plantean, por una parte, medidas para mitigar, adaptarse y corregir patrones de conducta a través de un marco regulatorio en materia ambiental y, de otra parte, generar un escenario propicio para a través de políticas que fomenten este tipo de innovaciones.

Con las innovaciones ambientales, se conciben productos, procesos y servicios que introducen mejores y solucionan problemas generando un valor compartido para stakeholders y shareholders; aportando a (i.) la reducción de los impactos negativos al ambiente, al mismo tiempo que se generan impactos positivos al mismo o (ii.) soluciones de blidad (Vargas-Chaves, 2016).

A su vez, la propiedad intelectual, como disciplina jurídica y de gestión de activos intangibles, ha comprendido la dinámica de la innovación como motor del bienestar y el desarrollo de la sociedad. Ello, gracias al reconocimiento de derechos que confieren la exclusividad a autores, inventores y titulares sobre sus obras, signos distintivos, obtenciones vegetales, diseños industriales, modelos de utilidad e invenciones, entre otras categorías de activos intangibles de propiedad intelectual.

Para el caso de las patentes, cuyo ámbito de aplicación se da sobre estos dos últimos activos, la exclusividad conferida por el Estado a favor de innovadores e inversores para explotar su producto o proceso, les da la potestad de controlar por un tiempo determinado el uso, goce y disposición de invenciones y modelos de utilidad, mientras esperan el retorno de su inversión y los ingresos que le supondrán una rentabilidad durante 10 o 20 años, según sea la figura escogida. En definitiva, y tal como se sostendrá en líneas posteriores, las patentes han contribuido notablemente a un crecimiento acelerado del desarrollo científico y a las transformaciones tecnológicas en diversas áreas del conocimiento e industrias. 
El presente artículo, desarrollado en el marco de una línea de investigación en biociencias y derecho en la Universidad Militar Nueva Granada (Colombia), busca poner de relieve, a través de una metodología basada en un razonamiento de corte deductivo, el rol de las patentes sobre aquellas innovaciones ambientales que se traducen en productos y procesos cobijados bajo las figuras de modelo de utilidad e invenciones. Para ello, en primer lugar presentaremos una serie de reflexiones sobre el ambiente y los recursos naturales en el proceso económico, a continuación ilustraremos nuestro punto de vista sobre la protección del ambiente como factor determinante para las innovaciones.

Lo anterior permitiendo abordar a continuación el status quo de la propiedad intelectual dentro de la innovación ambiental para así esbozar algunas reflexiones que nos permiten sostener que las patentes se han convertido en un eslabón clave para mejorar las referidas condiciones de deterioro ambiental y, al mismo tiempo, para fomentar la introducción de nuevos productos, procesos y servicios sostenibles y amigables con el ambiente. Por último, se presentan una serie de reflexiones finales a manera de conclusión.

\section{El ambiente y los recursos naturales en el proceso económico}

La apropiación de los recursos naturales por parte del ser humano ha sido una constante para su supervivencia desde la última gran glaciación y hasta el día de hoy para satisfacer otras necesidades (ÁngelMaya, 2013). Sin embargo, conforme empieza a construir su medio, el hombre ha pasado por alto aquellas leyes de la naturaleza a las que está sometido; leyes que regulan el equilibrio del ecosistema y de las cuales desde el inicio de su existencia como especie ha dependido.

Cuando finalmente comprendemos que la actividad de origen antropocéntrico puede alterar el equilibrio de todo nuestro entorno es que vemos la protección al ambiente como una necesidad imperante para la conservación de la especie humana. En efecto, el planeta en el que nos desenvolvemos como especie al tener el potencial de ser transformado por cualquier introducción tecnológica, tiene el potencial de genera una modificación del equilibrio local. 
Desde una óptica epistemológica, Ángel-Maya (2013) se refiere “a los sistemas tecnobiológicos sistemas artificiales construidos por la actividad humana [que] no siguen exactamente las leyes que rigen el ecosistema" (p. 50). Al respecto conviene destacar como el problema ambiental vendría dado no por la necesidad de 'conservar la naturaleza' a manera de reduccionismo ecológico, sino en modificarla de una forma adecuada, toda vez, que es el ser humano quien, como consecuencia de la misma evolución, debe aceptar su destino tecnológico.

Sin omitir ninguna de las dos ópticas -proteccionista y evolucionista- debe admitirse el hecho que los problemas ambientales vienen dados cuando el desarrollo tecnológico se desliga de las dinámicas del entorno sobre las cuales se afinca, alterando las cadenas tróficas y los ciclos biogeoquímicos, cuya velocidad de transformación amenaza la resiliencia misma de los ecosistemas y con ella la viabilidad de las sociedades y sectores productivos que dependen de la oferta de bienes y servicios que se desprenden de aquellos.

De allí que Georgescu-Roegen (2011) destacara el papel del ambiente y los recursos naturales en el proceso económico, al cuestionar el hecho de que la economía moderna esté atravesada por una epistemología mecanicista en la que se asume que la naturaleza funciona "de acuerdo con unas leyes mecánicas y [que] todas las cosas del mundo material podían explicarse en términos de la disposición y el movimiento de sus partes" (Capra, 1992, p. 63).

Ciertamente, ello estaría en la base de lo que Rifkin (1990) denomina aprieto entrópico de la humanidad, en el que la escasez económica toma dos direcciones dictadas por el carácter limitado de los recursos naturales y la irrevocable degradación entrópica. Ello desvirtúa la creencia económica convencional según la cual siempre se puede encontrar una solución, pase lo que pase.

Para comprender este punto, conviene recordar que la segunda ley de la termodinámica pone énfasis en la Entropía, entendida como el índice relativo de la energía no disponible en un sistema aislado, en los términos expuestos por Georgerscu-Roegen (citado por Rifkin, 1990, p. 301), quien introduce la concepción de irreversibilidad en los 
procesos de transformación de la energía, toda vez que "en cualquier proceso la energía tiende espontáneamente a asumir formas cada vez menos preciosas: tiende, como se dice, a degradarse, a descender en la escala de valores. [Si bien] su cantidad permanece igual, su calidad empeora" (Silvestrini, 1998, p. 21).

Ahora bien, en cuanto a las transformaciones de los ecosistemas, frecuentemente dadas con la intención de que los deseos y concepciones humanas sean adaptadas a la naturaleza, éstas reflejarían patrones no acordes con las capacidades mismas que éstos tienen para regenerarse, o lo que Holling (1973) dimensionó cuando abordó el concepto de resiliencia ecológica (Brand, 2009; Márquez, 2004).

La protección del ambiente como factor determinante para la innovación

Al violar los límites biológicos que le impone el entorno natural, la especie humana fue haciéndose más dependiente del uso de órganos exosomáticos (herramientas) cuyo funcionamiento es posible gracias a los recursos naturales, en especial los no renovables, mediado por un tipo de intercambio material y energético entre el hombre y la naturaleza que desencadena un proceso metabólico muy particular, donde el ser humano reproduce reiteradamente sus condiciones materiales y arroja sus características más distintivas en la fase actual de la economía global (Toledo, 2008).

En efecto, en la modernidad la causa de las trasformaciones no naturales devienen del tipo de economía que se viene desarrollando y que opera en dos ámbitos, sobre un mundo inerte como la industria metal-mecánica, y sobre el mundo vivo, que ha sufrido devastadores desajustes sociales, totalmente distintos a los se presentan como consecuencia de la evolución natural (Gómez-Giraldo, 2010).

Esto ha sido determinante para que en el ámbito internacional se diera, desde la década de los sesenta, un escenario de preocupación por las amenazas derivadas del modelo económico y los ritmos de apropiación, transformación, circulación, consumo y excreción del 
proceso metabólico prevaleciente, entendido como "los intercambios de flujos de materiales y energía, incluyendo el agua y los desechos, que todo país realiza con la naturaleza, es decir, en su entorno natural y con otros países" (Di Donato, 2010, p. 173).

Dichas preocupaciones se materializarían en puntos de inflexión del Derecho Internacional Ambiental como lo fue la Conferencia de Estocolmo de 1972, en la cual se llegó a una Declaración con principios (Nogueira, 2009). Destacando particularmente el principio cinco, según el cual, un correcto uso de los recursos no renovables representa una forma de afrontar el agotamiento de los mismos, mientras se otorgan garantías para que toda la humanidad pueda beneficiarse de ese buen manejo.

Esto a su vez fundamentaría desarrollos conceptuales posteriores como el contenido en el Informe 'Nuestro Futuro Común' -también conocido como Informe Brundtland- que fuera encargado por el Programa de las Naciones Unidas para el Medio Ambiente (PNUMA, 1983), cuya publicación en 1987 nos legó la noción de Desarrollo Sostenible entendido como aquel “(...) desarrollo que satisface las necesidades de la generación presente sin comprometer la capacidad de las generaciones futuras para satisfacer sus propias necesidades" (ONU, 1987).

Si nos atenemos al punto de partida epistemológico desde el cual se estructuran los presupuestos conceptuales de la Economía Ecológica, según la cual la economía es apenas un subsistema de uno mucho más grande pero finito - esto es la ecosfera- es posible inferir que la referida noción es una respuesta ante los desequilibrios que venían presentándose en el plano ecológico (Martínez-Alier \& Roca-Jusmet, 2015).

Siguiendo esta línea, Gómez-Giraldo (2005) es enfático en sostener que "(...) la naturaleza, puede subsistir sin nosotros, y de hecho el hombre es un fenómeno reciente en la evolución, mientras que él surge de ella y dependiendo irremediablemente de ella. Somos un apéndice del que el conjunto unitario puede prescindir, seguramente haciendo sus ajustes internos, pero nunca a la inversa" (p. 129). 
En efecto, asumiendo la definición de sistema dada por Ferdinand de Saussure hacia 1931, según la cual un sistema presenta un todo organizado, conformado de elementos solidarios que se unen en función de llegar a una totalidad (Hurtado, 2006). Las discusiones en torno a la protección del ambiente darían cuenta de un manejo de la crisis que supone las condiciones actuales en las cuales se desenvuelve el modelo económico (Rodríguez y Vargas-Chaves, 2016), siendo una respuesta a las falencias de la actividad estatal estabilizadora, por lo cual las crisis mismas son percibidas -bajo este enfoque-como el enfrentamiento de tendencias de desarrollo, donde el resultado es impredecible (Offe, 1990).

Al respecto, destacan los aportes hechos por teóricos del conflicto social tales como Coser (1956), Pellizzoni (2011) o Rodríguez (2016) quienes destacan que la vida en sociedad implica un devenir de cambios, lo cual, extrapolado a la discusión en torno a la crisis y a su manejo, conlleva a entenderlas como fuente creadora de nuevas formas o instituciones que estimulan el ámbito económico y tecnológico.

Justamente, en este intersticio es que reside la potencialidad inherente a la innovación en un marco de desarrollo económico, tal y como señalara Schumpeter, quien en alguna ocasión se refirió al impacto de los inventos y las innovaciones, evitando caer -desde lo acá propuesto- en "la actitud que ahora prevalece hacia el problema entrópico de la humanidad, la cual asume que de una u otra forma, la tecnología nos salvará de cualquier agujero en que podamos caer" (Georgescu-Roegen, 2011. p. 197). De allí la pertinencia que adquiere la conceptualización en torno a las innovaciones ambientales.

Y es que más allá de la innovación que, per se es entendida como "el proceso que entrelaza el conocimiento, los activos y las redes de empresas para transformar ideas, inventos en nuevos procesos, productos y servicios que capturen mercados (...)" (Ramírez-Vallejo, 2012 , p. 12), la razón de ser de la misma es su capacidad de incrementar los niveles de productividad como vía para elevar la competitividad de las empresas o de los países, y además las condiciones en que dicha productividad se inserta. 
Para el caso sub examine, se hace relevante su concepto en la adecuación al principio de desarrollo sostenible antes esbozado, en tanto que, lo que se propugna es evitar sobrepasar los umbrales de la resiliencia ecológica, asumida como la capacidad de un ecosistema de resistir un disturbio y aun así mantener su identidad específica (Brand, 2009).

Por lo demás, son válidos los postulados de Porter \& Stern (2001) sobre el papel que juega la presión y la adversidad sobre la capacidad de las empresas para innovar y, el -también-postulado de RamírezVallejo (2012), quien plantea un panorama de aproximación de la innovación ambiental al insertar consideraciones sobre la necesidad de que dichas innovaciones atiendan los desafíos que viene enfrentando esa ecosfera finita.

\section{La propiedad intelectual y la innovación ambiental}

Dentro de los objetivos estratégicos de la Organización Mundial de la Propiedad Intelectual (OMPI), destaca el "[abordar] la propiedad intelectual en relación con las cuestiones de política mundial”, lo cual ha marcado los derroteros de actuación de la organización, en tres frentes que responden a los desafíos globales: cambio climático, salud pública mundial y seguridad alimentaria (World Intellectual Property Organization, 2018).

En ese sentido, ha sido de interés que la Agenda de Desarrollo de este organismo internacional lleva a cabo acciones para implementar herramientas prácticas de propiedad intelectual que permitan generar verdaderas soluciones a esos retos que, en materia ambiental, nos demanda la sociedad y el planeta.

Por tal motivo, en el Programa y Presupuesto para 2012-2013, los Estados miembros de la OMPI (WIPO) ordenaron a este organismo internacional llevar a cabo acciones tendientes a la innovación abierta y la difusión de tecnologías verdes a través de una cadena de valor de la innovación de tecnología ecológica para comercializar, licenciar o acceder a una tecnología ecológica (World Intellectual Property Organization, 2018). 
En cuanto a los avances que se han dado en materia de innovación verde, según el informe "Global Challenges Report. Innovation and Diffusion of Green Technologies: The Role of Intellectual Property and Other Enabling Factors", la Organización Mundial de la Propiedad Intelectual-OMPI ha señalado que la debida protección de patentes juega un rol fundamental al momento de afrontar los retos de desarrollo tecnológico, difusión y financiamiento de este tipo de tecnologías.

Algunas de las más importantes innovaciones ambientales se despliegan en el sector de energía, al ser -junto con la agricultura, silvicultura y otros usos del suelo- el sector de mayores emisiones. En este sentido varias de las soluciones innovadoras están centradas en el desarrollo de fuentes alternativas de energía.

Es de resaltar que en el citado informe se hallan estadísticas de la segunda década del 2000 en países desarrollados respecto las solicitudes de patentes de innovaciones ambientales, las cuales estuvieron concentradas en 4 tipos de tecnología: biocombustibles, energía térmica solar, solar fotovoltaica y energía eólica, con un crecimiento de un $24 \%$ frente al 6\% que registraron otros tipos (World Intellectual Property Organization, 2015a).

Para el caso de Europa, resulta ilustrativa la tendencia que se ve en la Figura 1 y que muestra la Oficina de Patentes de Europa que en su informe "Climate change mitigation technologies in Europeevidence from patent and economic data", evidencia el aumento en las solicitudes de patentes de innovación verde o, específicamente Tecnologías de Mitigación del Cambio Climático. Ello, en un entorno en el que el aporte de las innovaciones tecnológicas en materia de mitigación y adaptación.

Esto, sin lugar a duda, representa una oportunidad para reducir la brecha antes referida, en un contexto en el cual la producción de innovaciones ha registrado crecimientos notorios que, para el caso de Europa pasó de una participación del 18\% al 40\%, siendo Alemania el país más innovador (European Patent Office, 2014). 


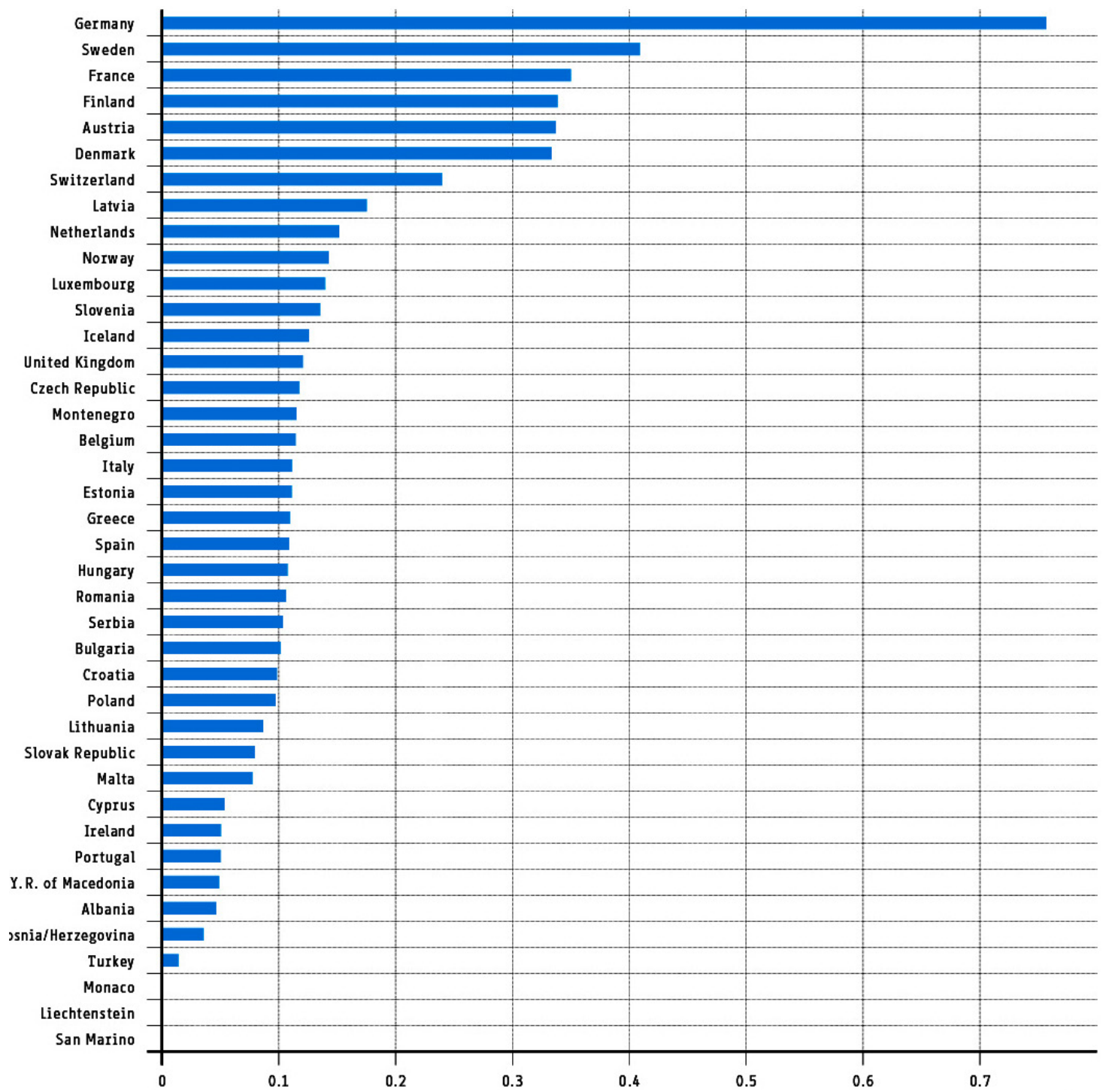

Figura 1. Evidencia de invenciones patentadas sobre tecnologías de mitigación del cambio climático (1995-2011). Fuente: European Patent Office (2014).

Por su parte, en el contexto latinoamericano y caribeño el panorama no es muy halagador. El más reciente informe de la Organización Mundial de la Propiedad Intelectual permite concluir que se tienen rezagos en materia de solicitud de patentes para innovaciones ambientales: menos del 3\% de las patentes solicitadas provienen de países de la región -tal y como se constató entre 1995 y 2011-, tan sólo por encima de las cifras que arroja África (World Intellectual Property Organization, 2015a). 
De hecho, en la Figura 2 se puede evidenciar como ninguno de los dos continentes figura en la 'Geografía de las innovaciones verdes' publicada por la Organización Mundial de la Propiedad Intelectual en el año 2015 (World Intellectual Property Organization, 2015b).
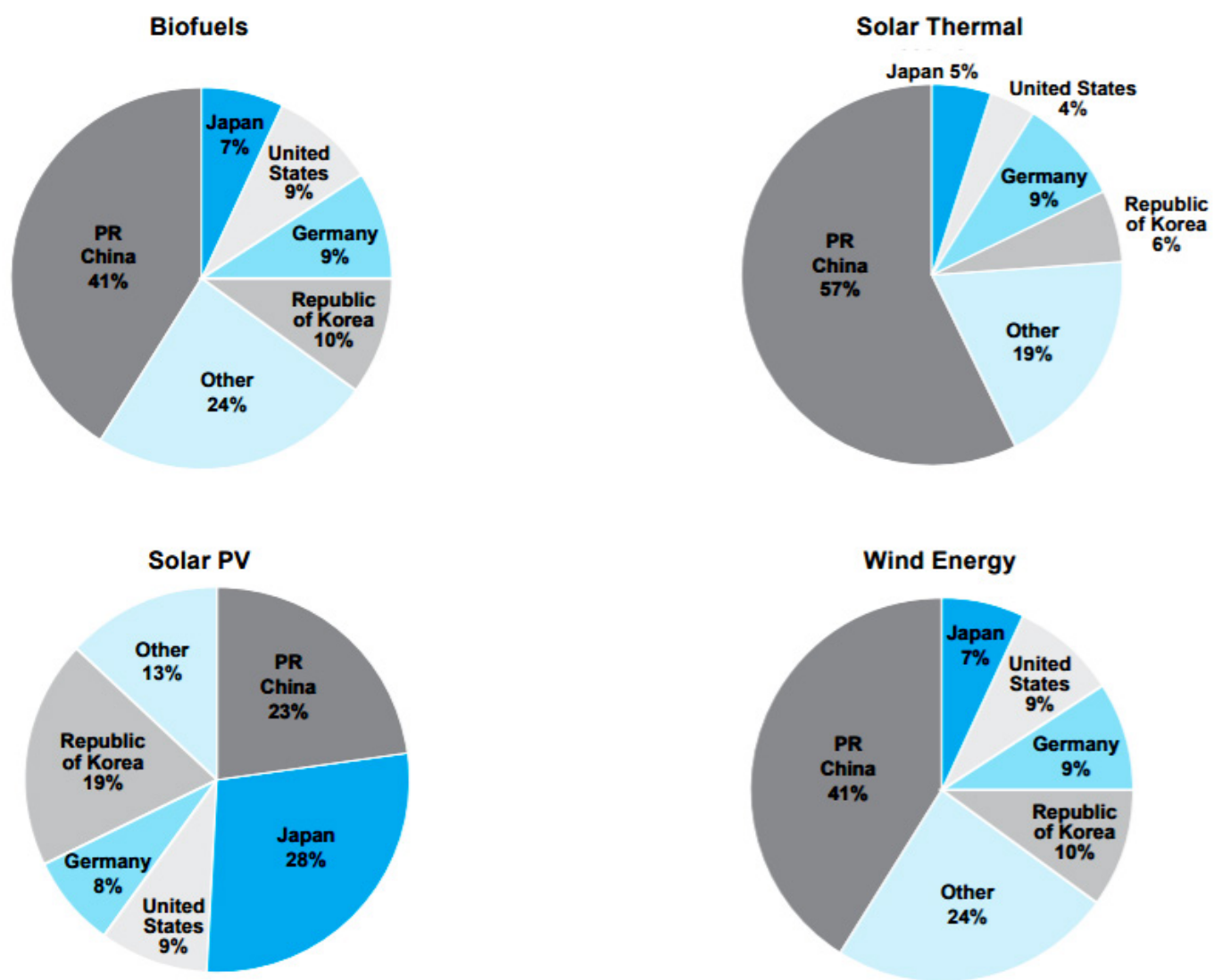

Figura 2. The geography of green innovation: patent application filings for four renewable energy technologies between 2006-2011.

Fuente: World Intellectual Property Organization (2015a).

En cualquier caso, es una realidad que esperamos cambie para la publicación de un próximo informe que, se estima, estará publicado en 2020. Allí, se verán reflejados los esfuerzos de los Estados miembros de la OMPI por impulsar la generación de productos, procesos y servicios que representen verdaderas soluciones a problemas como la contaminación, el uso desmedido de recursos naturales o el cambio climático (Helm, 2014). Ello, gracias a los incentivos y facilidades que despliegan los Estados para tramitar la solicitud de patentes. 
Así, por ejemplo, Australia, Israel, Canadá, China, Estados Unidos, Brasil, Corea, Japón, Reino Unido y España, por ejemplo, han logrado implementar un trámite de menor duración frente a las solicitudes referidas a tecnologías limpias (European Patent Office, 2014), con el fin de lograr su pronta comercialización y una mejor y mayor integración a las dinámicas propias de un mercado ávido de estas nuevas tecnologías.

\section{Las patentes como un eslabón clave del quehacer del experto en derecho y gestión ambiental}

La innovación como proceso integrado al desarrollo económico no se ha cultivado en un escenario de filantropía donde, tanto inversores como innovadores, se generan productos y procesos sin la expectativa de retorno de la inversión y rentabilidad. Es por esto que, la propiedad intelectual contempla como incentivo la exclusividad que le confiere el Estado a un innovador para explotar su producto o proceso, lo cual le permite controlar por un tiempo determinado el uso, goce y disposición de aquellos bienes inmateriales que surgen de su capacidad inventiva (Vargas-Chaves, 2016).

Las patentes como mecanismo de protección de las invenciones y modelos de utilidad, han contribuido a un crecimiento acelerado del desarrollo científico y a las transformaciones tecnológicas en diversas áreas del conocimiento e industrias como la del software o la industria farmacéutica (Correa, 2004; Smith \& Mann, 2004).

Al preguntarse por la pertinencia de las patentes como un eslabón de la propiedad intelectual, que a su vez es eje de la innovación ambiental, conviene anotar que los sistemas de propiedad intelectual que se han venido estructurando a su alrededor se han constituido en un elemento coadyuvante al beneficio común mediante el intercambio de conocimientos, por lo cual está llamada a contribuir en la conservación de los recursos naturales.

De allí que, que deban tenerse a las patentes como un (i.) instrumento que protege -a través de la referida exclusividad conferida- la propiedad [intelectual] privada; (ii.) una alternativa que fomente la introducción de nuevas tecnologías y procesos amigables 
con el ambiente y, (iii.) una herramienta que, además de entender los frutos del intelecto humano en una suerte de economía de las ideas, genera un valor añadido para la sociedad que previamente le ha conferido a estos productos cognitivos un rol como eslabón clave (Sádaba, 2008) que propugne por un equilibrio entre el bienestar de la sociedad y el mantener esos bienes y servicios que nos ofrece el ecosistema (Bermúdez-Guerrero, 2018).

Ezzi \& Jarboui (2016) al respecto se refieren al efecto de las estrategias de innovación -donde la patente juega un rol fundamental- en el desempeño financiero, social y ambiental desde el sector privado, y demuestran la existencia de una creciente preocupación por incorporar nuevos criterios al momento de innovar, los cuales no sólo atienden a los requerimientos de desempeño financiero y metas de productividad de las empresas, sino que tienen en cuenta variables como la ambiental, que también son determinantes al momento de hablar de competitividad.

En similar dirección Rehfeld, Rennings \& Ziegler (2007) evidencian cómo la innovación ambiental representa un verdadero proceso de innovación que permite abrir paso hace un real desarrollo sostenible; siempre y cuando se contemple un enfoque en el cual tengan cabida tanto la innovación tecnológica, como la social y la institucional. Esto es lo que se conoce como enfoque múltiple, desde el cual, autores como Duarte, Aranda \& Rata (2002), sitúan a la propiedad intelectual junto a la transferencia de tecnología como motores de los procesos de innovación tecnológica.

Considerando lo anteriormente expuesto, podemos entonces afirmar que las invenciones y modelos de utilidad patentados, no son sólo un activo de las empresas que contribuye a valorizarlas, son un motor en la generación de unas mejores condiciones en lo ambiental y social. Desde otra perspectiva, tienen este rol en tanto se dan las condiciones de la demanda asociada a la 'inteligencia verde' que tienen usuarios y consumidores al adquirir productos y servicios que contribuyan a un mejoramiento de dichas condiciones. 
Dentro de la teoría de la innovación ambiental las patentes contrarrestan el efecto de aquellos mercados que le dan prioridad a productos, procesos y servicios que representan una alta rentabilidad para los shareholders sin tener en cuenta el impacto ambiental que estas pueden llegar a producir (Rodríguez y Vargas-Chaves, 2015), puesto que al introducirse invenciones o mejoras de producto, proceso o servicio amigables con el ambiente y apalancados en la exclusividad que confiere la patente, se generan una nuevas dinámicas que refuerzan políticas e iniciativas que buscan generar conciencia al existir productos, procesos y servicios que suplen aquellos 'rentables'.

Cabe precisar que la innovación ambiental desde su teoría comprende:

Procesos, productos o procedimientos que [representan] un cambio que introduce novedades y que, a la vez, es capaz de generar un valor para el empresario, el consumidor y en general para toda la sociedad. Ello, en tanto es capaz de reducir impactos ambientales negativos o de generar impactos positivos; así como de fomentar, implementar o coadyuvar a la implementación de estrategias sostenibles en sus tres dimensiones: social, económica y ambiental (Vargas-Chaves, 2016, p. 21).

Retomando el planteamiento anterior, véase el caso de las bolsas plásticas que por sus bajos costos de producción representaban una rentabilidad a los supermercados: con la introducción de bolsas reutilizables con nuevos materiales -protegidos como procesos a través de patentes-, le plantearon al consumidor una alternativa que le facilitó al Estado implementar una política de uso racional de bolsas plásticas en supermercados.

En igual situación se encuentran los pitillos o pajillas, hoy reemplazados por una versión biodegradable patentada. No en vano, si no existiese la figura de la patente no existiría tampoco un incentivo para los ecoinnovadores que crearon este tipo de pitillos a partir de la experimentación con nuevos materiales (Epicoco, Oltra \& Saint, 
2014; Chulvi \& Vidal, 2011) pues no tendrían un retorno de la inversión ni tampoco aspirarían a generar una rentabilidad.

En suma, lo interesante del concepto de patentes como eslabón clave -como lo sugiere el título de este artículo- es el giro epistemológico que admite la innovación ambiental de la mano de la propiedad intelectual en la forma de concebir la gestión ambiental y la sostenibilidad en sus tres dimensiones -social, económica y ambiental- respondiendo a la función social y, al mismo tiempo, en las necesidades y expectativas de una sociedad con una visión -aún antropocéntrica- basada en las necesidades y aspiraciones del ser humanao.

\section{Conclusiones}

Las presiones sobre el entorno natural, los consabidos impactos sociales, económicos y ambientales derivados de una visión eminentemente antropocéntrica, amenazan no sólo el bienestar de la sociedad, sino que amenazan la rentabilidad del mismo sector que los origina. No en vano su productividad está relacionada de la interdependencia del ambiente con sus procesos y outputs.

Al mismo tiempo, la innovación ha adquirido progresivamente un mayor peso como vía para el diseño, formulación y puesta en marcha de soluciones o alternativas que mitiguen los efectos sobre el entorno, a partir de la generación de productos, procesos y servicios que generan impactos positivos al ambiente, mitiguen impactos negativos o que sean soluciones de sostenibilidad (Vargas-Chaves, 2016). Con la exclusividad conferida por las patentes, la innovación y el desarrollo son ya por si mismos incentivos si se piensa incluso sólo en términos de rentabilidad.

Al igual que otros incentivos dirigidos a fomentar la responsabilidad ambiental de las empresas, las patentes sobre innovaciones ambientales están llamadas a jugar un rol clave en las políticas ambientales. El valor compartido que se genera para stakeholders y shareholders con la exclusividad temporal conferida por la patente y con su posterior acceso con el dominio público, han sido además claves en este sentido. 
Pese a ello, debemos reconocer que esto no es la solución definitiva a las condiciones de deterioro ambiental ocasionadas por el hombre desde la industrialización. Además de un cambio de mentalidad, los Estados deben contemplar reforzar mecanismos tales como subvenciones, impuestos a las tecnologías contaminantes, impuestos a las emisiones de gases de efecto invernadero, entre otros instrumentos; impuestos que incluso pueden utilizarse para fomentar el desarrollo de nuevas tecnologías.

\section{REFERENCIAS}

Ángel-Maya, A. (2013). El reto de la vida. Ecosistema y Cultura. Una introducción al estudio del Medio Ambiente. (2 ed.). Bogotá, D.C.: Ecofondo. Disponible en https://rds.org.co/apc-aa-files/ ba03645a7c069b5ed406f13122a61c07/el_reto_de_la_vida. pdf

Bermúdez-Guerrero, O. M. (2018). Educación Ambiental, valores y prácticas sustentables. Una guía para Educadores del Siglo XXI. Bogotá. D.C.: Universidad Nacional de Colombia.

Brand, F. (2009). Critical natural capital revisited: Ecological resilience and sustainable development. Ecological Economics, 68(3), 605-612. https://doi.org/10.1016/j.ecolecon.2008.09.013

Capra, F. (1992). El punto crucial. Ciencia, sociedad y cultura. Buenos Aires: Troquel.

Chulvi, V. \& Vidal, R. (2011). Usefulness of evolution lines in ecodesign. Procedia Engineering, 9(1), 135-144. https://doi. org/10.1016/j.proeng.2011.03.107

Correa, C. (2004). Ownership of knowledge: the role of patents in pharmaceutical R\&D. Bulletin of the World Health Organization, 82(1), 784-787. Available from https://www.ncbi. nlm.nih.gov/pmc/articles/PMC2623027/

Coser, L. A. (1956). The functions of social conflict. London: Routledge. 
Di Donato, M. (2010). Entrevista a Víctor M. Toledo: 'La crisis de civilización de la humanidades una crisis de relaciones de la sociedad industrial con los procesos naturales'. Papeles de relaciones eco-sociales y cambio global, (110), 171-177.

Duarte, A. R., Aranda, D. A. y Rata, B. M. (2002). Un marco de análisis para la innovación tecnológica en la empresa. $D i$ rección y Organización, (27), 25-36.

Epicoco, M., Oltra, V. \& Saint Jean, M. (2014). Knowledge dynamics and sources of eco-innovation: Mapping the Green Chemistry community. Technological Forecasting and Social Change, 81(1), 388-402. https://doi.org/10.1016/j.techfore.2013.03.006

European Patent Office. (2014). Climate change mitigation technologies in Europe - evidence from patent and economic data. [Online]. Recuperado de https://www.epo.org/mobile/newsissues/technology/sustainable-technologies/clean-energy/ europe.html

Ezzi, F. \& Jarboui, A. (2016). Does innovation strategy affect financial, social and environmental performance? Journal of Economics, Finance and Administrative Science, 21(40), 14-24. https://doi.org/10.1016/j.jefas.2016.03.001

Georgescu-Roegen, N. (2011). ¿Qué puede enseñar a los economistas la termodinámica y la biología? En, F. Aguilera \& V. Alcántara, (Eds.). De la economía ambiental a la economía ecológica (pp. 188-198). Barcelona: ICARIA. Disponible en https://economiaecologicaunam.files.wordpress. com/2014/09/n-georgescu-roegen.pdf

Gómez-Giraldo, L. (2010). Economía ecológica. Bases operativas: una ecopolitica. Bogotá, D.C.: Universidad Nacional de Colombia.

Gómez-Giraldo, L. (2005). El concepto de sostenibilidad ecológica: génesis y limites. Bogotá, D.C.: Universidad Nacional de Colombia. 
Helm, D. (2014). The European framework for energy and climate policies. Energy Policy, 64(1), 29-35. https://doi.org/10.1016/j. enpol.2013.05.063

Holling, C. S. (1973). Resilience and stability of ecological systems. Annual Review of Ecology and Systematics, 4(1), 1-23. https://doi.org/10.1146/annurev.es.04.110173.000245

Hurtado, S. (2006). Justicia, políticas públicas y bienestar social. México, D.F.: Universidad Nacional Autónoma de México.

Márquez, G. (2004). Mapas de un fracaso. Naturaleza y conflicto en Colombia. Bogotá, D.C.: Universidad Nacional de Colombia. Martínez-Alier, J., \& Roca-Jusmet, J. (2015). Economía ecológica y política ambiental. México, D.F.: Fondo de Cultura económica.

Nogueira, P. (2009). A conferência de Estocolmo como ponto de partida para a proteção internacional do meio ambiente. Revista Direitos Fundamentais \& Democracia, 6(6). 1-25.

Offe, C. (1990). Crisis en el manejo de la crisis. Elementos para una teoría de la crisis política. En, J. Keane, (Ed.). Contradicciones en el Estado de Bienestar (pp. 288-309). Madrid: Alianza.

ONU. (1987). Informe de la Comisión Mundial sobre el Medio Ambiente y el Desarrollo. [Online]. Recuperado de http://www. ecominga.uqam.ca/PDF/BIBLIOGRAPHIE/GUIDE_LECTURE_1/CMMAD-Informe-Comision-Brundtland-sobreMedio-Ambiente-Desarrollo.pdf

Pellizzoni, L. (2011). The politics of facts: local environmental conflicts and expertise. Environmental Politics, 20(6), 765-785. https://doi.org/10.1080/09644016.2011.617164

Porter, M. E. \& Stern, S. (2001). Innovation: location matters. MIT Sloan management review, 42(4), 28-28.

Ramírez-Vallejo, J. (2012). Clúster, una visión alternativa para el desarrollo alternativo. En, M. Rodríguez-Delgado (Ed.). Gestión de clúster en Colombia: una herramienta para la competitividad (pp. 78-94). Bogotá. D.C.: Universidad de los Andes. 
Rehfeld, K. M., Rennings, K. \& Ziegler, A. (2007). Integrated product policy and environmental product innovations: An empirical analysis. Ecological economics, 61(1), 91-100. https:// doi.org/10.1016/j.ecolecon.2006.02.003

Rifkin, J. (1990). Entropía. Hacia el mundo invernadero. Barcelona: Urano.

Rodríguez, G. A. y Vargas-Chaves, I. (2016). La prevención en materia ambiental: tendencias actuales. Bogotá, D.C.: Universidad del Rosario.

Rodríguez, G. A. y Vargas-Chaves, I. (2015). Perspectivas de responsabilidad por daños ambientales en Colombia. Bogotá. D.C.: Editorial Universidad del Rosario.

Rodríguez, G. A. (2016). Los conflictos ambientales en Colombia y su incidencia en los territorios indígenas. Bogotá, D.C.: Editorial Universidad del Rosario.

Sádaba, I. (2008). Propiedad intelectual: ¿̇bienes públicos o mercancías privadas? Madrid: Los libros de la Catarata.

Silvestrini, V. (1998). ¿Qué es la entropía? Bogotá, D.C.: Norma.

Smith, B. L. \& Mann, S. O. (2004). Innovation and Intellectual Property Protection in the Software Industry: An Emerging Role for Patents? The University of Chicago Law Review, 71(1), 241-264.

Toledo, V. M. (2008). Metabolismos rurales: hacia una teoría económica-ecológica de la apropiación de la naturaleza. Revista Iberoamericana de Economía Ecológica, 7(1), 1-26.

Vargas-Chaves, I. (2016). Derecho e innovación ambiental. Bogotá, D.C.: Universidad del Rosario. http://dx.doi.org/10.12804/ ga9789587387469

World Intellectual Property Organization. (2018). La OMPI y los Objetivos de Desarrollo Sostenible. [Online]. Recuperado de https://www.wipo.int/publications/es/details.jsp?id=4354

World Intellectual Property Organization. (2015a). Global Challenges Report. Innovation and Diffusion of Green Technologies: The Role of Intellectual Property and Other. Ginebra: World Intellectual Property Organization. 
World Intellectual Property Organization. (2015b). Enabling Factors. [Online]. Recuperado de https://www.wipo.int/edocs/ pubdocs/en/wipo_rep_gc_2015_1.pdf

Iván Vargas-Chaves es Abogado de la Universidad del Rosario. Máster en Derecho de la Universidad de Génova (Italia). Máster en Derecho Privado de las Universidades de Salamanca (España), Pública de Navarra (España) y de Venecia Ca' Foscari (Italia). Doctor en Derecho de la Universidad de Barcelona (España). Doctor en Derecho Supranacional e Interno de la Universidad de Palermo (Italia). Profesor de carrera vinculado de tiempo completo a la Facultad de Derecho de la Universidad Militar Nueva Granada (Bogotá, Colombia). https://orcid.org/0000-0001-6597-2335

Omar Clavijo-Bernal es Politólogo de la Universidad Nacional de Colombia. Especialista en Derecho Ambiental de la Universidad del Rosario. Magíster en Medio Ambiente y Desarrollo de la Universidad Nacional de Colombia. Profesor de la Universidad Nacional de Colombia. https://orcid.org/0000-0003-0729-8228

Jonathan Sánchez-Rippe es Ingeniero Químico de la Universidad de los Andes (Colombia) y Especialista en Derecho Ambiental de la Universidad del Rosario (Colombia). https://orcid.org/0000-00031539-2492 